http://jmscr.igmpublication.org/home/ ISSN (e)-2347-176x ISSN (p) 2455-0450 crossref DOI: https://dx.doi.org/10.18535/jmscr/v8i6.60

\author{
Dournal Of Medical Science And Clinical Research \\ IGM Publication \\ An Official Publication of IGM Publication
}

\title{
To determine the distribution and nature of cranial MR imaging findings in patients with severe preeclampsia \& eclampsia and to evaluate their correlation with clinical and laboratory data: A study from North Indian tertiary health care institution
}

Authors

\author{
Dr Rama Thakur ${ }^{1}$, Dr Rohit Dogra ${ }^{2 *}$, Dr Vijay Thakur ${ }^{3}$ \\ ${ }^{1}$ Professor Department of Obstetrics and Gynecology Kamla Nehru Hospital Shimla \\ ${ }^{2}$ Department of Obstetrics and Gynecology Civil Hospital Jaisinghpur \\ ${ }^{3}$ Professor Department of Radiology IGMC Shimla H.P \\ *Corresponding Author
}

Dr Rohit Dogra

\begin{abstract}
Introduction: Hypertensive disorders of pregnancy comprising of pre-eclampsia and eclampsia are a major cause of adverse pregnancy outcomes. Neurological manifestations of pregnancy induced hypertension are the most common cause of maternal and foetal morbidity and mortality. Cranial neuroimaging reveals focal regions of symmetric hemispheric oedema; with parietal and occipital regions getting most commonly affected.
\end{abstract}

Methods: The study was conducted among 65 antenatal women diagnosed with pre-eclampsia and eclampsia at gestational age >20 weeks in the Department of Obstetrics and Gynaecology, Kamla Nehru State Hospital for Mother and child IGMC Shimla. Clinical signs and symptoms, Laboratory tests neuroimaging findings were recorded for study purpose.

Results: About 17.6\% of severe pre-eclampsia and 100\% of eclampsia had findings observed on cranial MRI. Headache and visual complaints were most frequently recorded. PRES was the predominant neuroradiographic finding in present study and occipital lobe was commonly affected region. In subjects with MR imaging findings, hematocrit, LDH, serum uric acid and ApTT were significantly higher than those without MR imaging findings. Subjects with MRI finding had abnormal RBC morphology whereas none of MR negative subjects had abnormal RBC morphology. No significant difference was observed regarding blood pressure parameters between MRI positive and negative subjects.

Conclusion: Neuroimaging in antenatals with severe hypertensive disorders might aid in better understanding of the poorly explained phenomenon. In addition this would be helpful in better management of the disorders along with their much dreaded complications. Patients with hypertensive disorders of pregnancy with deranged biochemical tets should be subjected routinely to cranial imaging for the better perinatal outcomes.

Keywords: Antenatal women, Pregnancy induced hypertension, Biochemical tests, neuro-imaging.

\section{Introduction}

Hypertensive disorders of pregnancy comprising of pre-eclampsia and eclampsia are a major cause of perinatal adverse outcomes both in terms of adverse maternal and foetal outcomes. ${ }^{1}$ These are the most common medical complications of pregnancy, with a reported incidence ranging between $6 \%$ and $8 \% .^{2}$ They are an important cause of severe morbidity and mortality among mothers and babies. In Africa and Asia, 
hypertensive disorders of pregnancy, especially eclampsia, are associated with nearly one-tenth of all maternal deaths. ${ }^{3}$

Despite availability of intensive care unit and improved antenatal care still some women die of eclampsia. The most common cause of death is cerebral complications. ${ }^{4} \quad$ Neurological manifestations of pregnancy induced hypertension vary from diffuse symptoms such as headache and confusion to focal signs such as paralysis and visual loss. Computerized tomography (CT) Scan and magnetic resonance imaging (MRI) have greatly enhanced our understanding of the correlation between neurological complaints and neuroanatomic pathological changes characteristic of Preeclampsia (PE) and Eclampsia (E). ${ }^{5}$ Cerebral microcirculation is the major target. 6 Acute fluctuation of blood pressure leads to failure of cerebral autoregulation. In response to acute and severe hypertension, cerebrovascular overregulation leads to vasospasm resulting in ischemia, cytotoxic edema and eventually tissue infarction.

Posterior reversible encephalopathy syndrome (PRES) refers to a clinical and radiological entity presented by headache, altered mental state such as confusion, lethargy, cortical visual disturbances and seizures with transient edematous changes of subcortical white matter on neuroimaging.7 Neuro-Imaging of brain typically demonstrates focal regions of symmetric hemispheric oedema, parietal and occipital lobes are commonly affected followed by the frontal lobes, inferior temporal occipital junction and the cerebellum. 8 Eclampsia patients who are refractory to $\mathrm{MgSo} 4$ and antihypertensive therapy have significant CNS pathology. 4

RBC morphology is the strongest predictor of abnormal radiographic findings. The only laboratory parameter that has been found to be abnormal a week prior to the development of neurological symptoms is serum Lactate dehydrogenase (LDH) level which is higher in the group that later developed hypertensive encephalopathy related brain oedema ${ }^{16}$. Alteration in Liver function tests (LFTs) includes elevated levels of serum aminotransferase, namely, aspartate aminotransferase (AST) or alanine aminotransferase (ALT). Renal perfusion and glomerular filtration rate are reduced. Plasma uric acid concentration is typically elevated in preeclampsia. The elevation exceeds the reduction in glomerular filtration rate and likely is also due to enhanced tubular reabsorption ${ }^{17}$.

Although several studies have been conducted to correlate the various clinical and laboratory parameters with abnormalities on neuro-imaging, yet only a few studies provide information on predictors of outcome in eclamptic encephalopathy. ${ }^{9}$ Keeping all the considerations in mind and dearth of such studies in the region, the present study was conducted with a purpose to determine the spectrum of neuro-Imaging findings in patients with severe preeclampsia and eclampsia.

\section{Materials and Methods}

Study Area and Population: The study was conducted among the pregnant women attending antenatal clinic in the Department of Obstetrics and Gynaecology, Kamla Nehru State Hospital for Mother and child IGMC Shimla.

Study Design: Descriptive observational study.

Study Period: One year from July 2016 to June 2017.

Study Sample: As it was a time bound study, a total of 65 subjects (preeclampsia and eclampsia patients) were included in study.

Inclusion and Exclusion Criteria: Pregnant women diagnosed with pre-eclampsia and eclampsia at gestational age $>20$ weeks singleton pregnancy were included in the study while women with essential hypertension, liver disease, septicemia cortical vein thrombosis, tuberculoma, neurological infections, epilepsy, encephalitis, brain abscess, neurological tumors and cerebral malaria were excluded.

Study Tool: A study proforma was designed to collect and record socio-demographic parameters, 
antenatal history, previous medical and reproductive history, clinical signs and symptoms, laboratory investigations, ultrasonic findings, neuroimaging findings, plan and mode of delivery and maternal/foetal outcomes were recorded on the proforma.

Methodology: Standard case definitions were utilized to identify study participants. Patients with severe preeclampsia/eclampsia at 20 weeks or beyond were enrolled for this study. On admission detailed history was taken including warning signs and symptoms i.e. headache, blurring of vision, visual field scotomas, blindness, nausea/vomiting, pain epigastrium swelling feet. Time of onset of seizure, duration \& type of seizure: tonic clonic movement, uprolling of eyeballs, frothing from mouth, deviation of head, post seizure confusion, number of seizures and any focal neurological deficit were also noted. These patients were managed in the emergency set up in labour room.

Sociodemographic variables were recorded on proforma, which was followed by general physical examination, obstetrical examination and biochemical analysis. Renal sonogram was done in subjects with chronic hypertension and decreased urinary output. All subjects enrolled for this study received antihypertensives and seizures prophylaxis with Magnesium Sulphate (Pritchards Regimen). Subjects at gestation $<34$ weeks received antenatal glucocorticoids for fetal pulmonary maturity. Pelvic and cervical assessment was done after stabilizing the patient and mode of delivery was decided accordingly. Caesarean section was done for the obstetric and medical indications only. Labour was monitored partographically. Second stage was cut short by prophylactic outlet forceps/ventouse. Labour and Neonatal parameters were recorded according to the performa.

Subjects with eclampsia, refractory eclampsia and severe preeclampsia with neurological symptoms severe enough to prompt neuroimaging were subjected to cranial MR imaging on 1.5 Tesla Magnetic Resonance Imaging system in
Department of Radiodiagnosis at IGMC Shimla within 4 days of presentation. The standard protocol comprised of T1 Weighted images, T2 Weighted images, FLAIR sequence along with Diffusion Weighted images. Vasogenicedema which is the characteristic finding in PRES, showed hypo-intense signal on T1WI, hyperintense signal on T2WI and FLAIR sequences and there was no diffusion restriction on Diffusion weighted images, whereas cerebral infarcts had shown diffusion restriction. The nature \& distribution of the cerebral lesions, if any was noted. The clinical findings, laboratory data of all the patients with or without radiological findings on cranial MR imaging was compared and correlated.

Statistical Analysis: Data were entered into Microsoft Excel spreadsheet, cleaned and transferred to Epi Info version 7.2.2.6 software for analysis. Continuous variables were presented as mean scores \pm standard deviations while discrete variables as percentages and proportions of each. Pearson's Chi-squared was used to test the statistical significance of categorical data respectively. Mean of variables was compared using Independent $t$-test after checking normality of data. Two tailed $P$ value $<0.05$ was considered as statistically significant for all analysis.

Ethical Considerations: Prior permission was taken from Institute Ethical Committee. Personal identifiers were omitted in order to maintain confidentiality and anonymity. Potential harms and benefits were explained to the patient and guardian before taking consent. Patient was free to leave the study at any point of time and this didn't affect her clinical care. No financial expenditure was incurred by the patient for the sake of study.

\section{Results}

The study recorded the findings of 65 Preeclamptic/Eclamptic antenatal women with gestational age more than 20 weeks and presenting with neurological symptoms. All 65 patients enrolled for the study were subjected to cranial MR Imaging. 
Table 1 shows the radiological findings of the subjects on Magnetic Resonance Imaging (MRI). About $36.9 \%$ subjects had findings on MRI. Posterior Reversible Encephalopathy Syndrome (PRES) was the most common (27.7\%) finding followed by PRES with vasospasm (3.1\%). One patient had radiological diagnosis of maxillary sinusitis on MRI and it was also regarded as MRI negative for the data analysis as the MRI of the brain was normal in this patient. About $63 \%$ of participants had no abnormality detected on MRI.

Table 1: Radiological Findings on Cranial Magnetic Resonance Imaging Findings Number (\% age)

\begin{tabular}{|l|c|c|}
\hline Findings & No. & Percentage \\
\hline Posterior reversible encephalopathy syndrome & 18 & $27.69 \%$ \\
\hline Posterior reversible encephalopathy syndrome with vasospasm & 2 & $3.07 \%$ \\
\hline Infarct & 1 & $1.54 \%$ \\
\hline CVT without infarct & 0 & $0 \%$ \\
\hline Atrophic changes & 1 & $1.54 \%$ \\
\hline Vasospasm & 1 & $1.54 \%$ \\
\hline Maxillary sinusitis & 1 & $1.54 \%$ \\
\hline No abnormality detected & 41 & $63.07 \%$ \\
\hline Total & 65 & \\
\hline
\end{tabular}

CVT: Cortical venous thrombosis

Table 2 depicts that 61 subjects had headache as the premonitory symptoms. Of these 18 had evidence of neurological involvement on MRI and the affected hemisphere region was occipital lobe. In total 14 subjects each had presented with seizures and disorientation; and they had evidence of neurological involvement on cranial MRI. In both scenarios, the affected hemisphere region was parieto-occipital lobes. About 52 subjects complained of blurring of vision, out of these 16 had evidence of neurological involvement and the lesion was located in occipital lobe in all of them. The subjects presenting with symptoms of vertigo and dizziness didn't show neurological involvement on MRI. Furthermore, it was observed that out of 23 subjects having lesions on MRI, $21 \quad(91.3 \%)$ subjects had bilateral hemispheric involvement and only two $(8.7 \%)$ subjects had unilateral hemispheric involvement.

Table 2: Relation of Neurological Symptoms with location of lesion on Neuroimaging

\begin{tabular}{|l|c|c|c|}
\hline Neurological symptoms & Frequency & Location on MRI & Frequency \\
\hline Headache & 61 & Occipital & 18 \\
\hline Seizure & 14 & Occipital \&Parietal & 14 \\
\hline Disorientation & 14 & Occipital \& Parietal & 14 \\
\hline Blurring vision & 52 & Occipital & 16 \\
\hline Vertigo & 0 & None & 0 \\
\hline Dizziness & 0 & None & 0 \\
\hline
\end{tabular}

Table 3 highlights the relation of MRI findings with various maternal parameters. Except less than 20 years age group there was no statistically significant difference pertaining to neurological findings. There was no statistically significant difference regarding MRI findings among different parity groups. Furthermore, only in 34 to 36 weeks 6 days gestational age group the difference was found statistically significant, where 6 subjects had neuroimaging findings.
Among symptoms, seizure disorder and depression of consciousness were the presenting symptoms where difference was significant ( $\mathrm{p}$ value $<0.001)$.

Out of 51 severe preeclampsia subjects, only 9 were MRI positive while among 14 subjects who presented with eclampsia all were MRI positive for neurological findings. The MRI positivity was statistically significant in those subjects who had Systolic Blood Pressure (SBP) in the range of 
140-159 mm, 160-190 mm and > $200 \mathrm{~mm}$ of $\mathrm{Hg}$. Similarly The MRI positivity was statistically significant in those subjects who had diastolic blood pressure in the range of 111-130 and > 130 $\mathrm{mm}$ of $\mathrm{Hg}$. The MAP of $\geq 105 \mathrm{~mm}$ of $\mathrm{Hg}$ was significantly associated with positive MRI findings. No significant difference was observed in presence or absence of neurological finding on MRI in relation to mean systolic, mean diastolic and mean arterial pressure

Table 3: Relation of MRI Findings with Maternal variables

\begin{tabular}{|l|c|c|c|c|}
\hline Parameter & Sub Group & MRI positive $(\mathrm{n}=23) \%$ & MRI Negative $(\mathrm{n}=42) \%$ & P-Value \\
\hline \multirow{4}{*}{ maternal age } & $<20$ & $3(13.1)$ & 0 & $0.016^{*}$ \\
\cline { 2 - 5 } & $20-29$ & $15(65.2)$ & $35(83.3)$ & 0.097 \\
\cline { 2 - 5 } & $30-39$ & $3(13.1$ & $7(16.6)$ & 0.69 \\
\cline { 2 - 5 } & $>40$ & $2(8.7)$ & 0 & 0.052 \\
\cline { 2 - 5 } & Mean \pm SD & $26.39 \pm 7.372$ & $24.48 \pm 3.486$ & 0.17 \\
\hline Gravidity & Primigravida & $11(47.8)$ & $27(64.3)$ & 0.19 \\
\hline & multigravida & $11(47.8)$ & $15(35.7)$ & 0.34 \\
\hline gestational age & postpartum & $1(4.3)$ & 0 & 0.17 \\
\hline & $20-23$ wks 6 days & $1(4.4)$ & $2(4.8)$ & 0.09 \\
\hline & $24-27$ wks 6 days & $4(17.4)$ & $10(23.7)$ & 0.83 \\
\hline & $28-33$ wks 6 days & $6(26.1)$ & $22(52.3)$ & $0.04^{*}$ \\
\hline & $34-36$ wks 6 days & $6(26.1)$ & $8(19.1)$ & 0.79 \\
\hline
\end{tabular}

Table 4 depicts the various laboratory parameters of subjects with severe preeclampsia/eclampsia and their correlation with MRI findings. Several lab parameters differed between MRI positive and MRI negative subjects. The difference was statistically significant for Hct (p-value 0.008), platelet count ( $p$-value 0.023), serum LDH (pvalue 0.0 ), S. uric acid( $p$-value 0.041), APTT( p- value 0.002 ) and abnormal red cell picture ( $p$ value 0.0 ). Rest of the lab parameters didn't differ significantly in relation to MRI findings ( $\mathrm{p}$-value $\geq 0.05)$. The mean thrombocyte count $(164739+/-$ $103060 / \mu \mathrm{l})$ was significantly higher in MRI positive subjects, the possible explanation for this unusual finding could be that the subject with HELLP and DIC were excluded from the study.

Table 4: Relation of Various Lab Parameters with MRI Findings

\begin{tabular}{|c|c|c|c|c|c|c|c|}
\hline \multirow{2}{*}{$\begin{array}{l}\text { LAB } \\
\text { PARAMETERS }\end{array}$} & \multicolumn{3}{|c|}{ MRI negative } & \multicolumn{3}{|c|}{ MRI positive } & \multirow[t]{2}{*}{ p-vale } \\
\hline & Minimum & Maximum & Mean+/-SD & Minimum & Maximum & Mean+/-SD & \\
\hline $\operatorname{Hct}(\%)$ & 23.2 & 42 & $35.830+/-5.213$ & 32 & 45 & $39.18+/-2.93$ & $0.008^{*}$ \\
\hline WBC $\left(10^{3} / \mathrm{ul}\right)$ & 9500 & 19880 & $14883+/-2641$ & 4900 & 19300 & $14226+/-3892$ & 0.423 \\
\hline 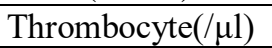 & 50000 & 238000 & $101269+/-46382$ & 50000 & 357000 & $164739+/-103060$ & $0.023^{*}$ \\
\hline $\mathrm{LDH}(\mathrm{U} / \mathrm{L})$ & 344 & 800 & $567+/-122.03$ & 479 & 1840 & $947.17+/-338.5$ & $0.00^{*}$ \\
\hline AST (U/L) & 33 & 234 & $72+/-58.8$ & 28 & 234 & $75.87+/-68$ & 0.823 \\
\hline ALT (U/L) & 18 & 334 & $85.86+/-84.4$ & 16 & 334 & $88.3+/-111$ & 0.921 \\
\hline ALP(U/L) & 120 & 490 & $238+/-81.09$ & 66 & 547 & $222+/-132$ & 0.550 \\
\hline Uric acid(mg/dl) & 4 & 8.6 & $6.102+/-0.913$ & 3.7 & 9.2 & $6.870+/-1.58$ & $0.041^{*}$ \\
\hline BUN (mg/dl) & 8.5 & 22.0 & $12.683+/-2.77$ & 7 & 24.5 & $13.75+/-5.26$ & 0.287 \\
\hline Creatinine(mg/dl) & 0.6 & 1.6 & $0.93+/-0.189$ & 0.7 & 1.5 & $1.024+/-0.27$ & 0.32 \\
\hline $\mathrm{TSP}(\mathrm{g} / \mathrm{dl})$ & 5 & 6.6 & $5.61+/-0.551$ & 4.7 & 7 & $5.79+/-0.7$ & 0.243 \\
\hline $\operatorname{Albumin}(\mathrm{g} / \mathrm{dl})$ & 2 & 5 & $3.298+/-0.722$ & 2.2 & 6 & $3.45+/-0.82$ & 0.491 \\
\hline PT(seconds) & 10 & 12.8 & $11.35+/-0.642$ & 10 & 13.5 & $11.787+/-1.25$ & 0.071 \\
\hline APTT (seconds) & 28 & 34 & $31.919+/-1.32$ & 30.6 & 39.2 & $34.109+/-2.98$ & $0.002^{*}$ \\
\hline $\begin{array}{l}24 \text { hrs urine } \\
\text { protein(mg) }\end{array}$ & 200 & 6468 & $596+/-1332$ & 220 & 1000 & $487+/-226.6$ & 0.700 \\
\hline $\begin{array}{l}\text { Abnormal red cell } \\
\text { picture(No.) }\end{array}$ & & & 0 & & & 16 & $0.00^{*}$ \\
\hline
\end{tabular}




\section{Discussion}

The present study highlights the neuro-imaging findings in the antenatal cases suffering from hypertensive disorders of pregnancy. The mean age in the MRI positive subjects in the present study was $26.39 \pm 7.37$ years which is comparable to that observed by Junewar V et al $(24.44 \pm 3.53$ years). ${ }^{9}$ No significant difference in maternal age in relation to MRI findings was observed. About $34.3 \%$ subjects were MRI positive. Furthermore 47.8\% MRI positive subjects were primigravidae, which was in contrary with the study conducted by Sharma $G$ et al where this proportion was $70.8 \% .^{10}$

In the present study $78.5 \%$ patients with severe preeclampsia were subjected for MR imaging with $17.6 \%$ being MRI positive. This was comparable to the study conducted by Schwartz RB et al and Sharma $\mathrm{G}$ et al where $<30 \% \%$ subjects of severe preeclampsia were MR imaging positive. ${ }^{11,12}$ This could be due to the fact that the subjects with severe preeclampsia were terminated at priority in order to halt the endothelial dysfunction and further neuropathology.

Timely termination of pregnancy and specialized care provided to such patients could be the possible explanation for the normal neuroimaging report in subjects with severe preeclampsia. A total of 14 eclamptic patients were subjected to neuroimaging, all having MRI findings. Similarly in study by Sharma G et al, all 21 subjects with eclampsia had positive MRI findings. ${ }^{12}$ These findings were consistent with diagnosis of PRES in majority of subjects in both the studies. The radiological findings in subjects with eclampsia in the present study had shown varying degree of hemorrhage, cerebral edema and vasculopathy. Schwartz RB et al also observed that the tonic clonic seizures were significantly more common in the group with radiographic abnormalities than in the group without such findings. ${ }^{11}$

MRI finding positive subjects who presented with headache and visual disorders were located in occipital lobe in the present study, which was in accordance with Dhayia $\mathrm{K}$ et al. $^{13}$ On neuroimaging, the majority of subjects had parietal and occipital lobe involvement, which was similar to findings of Schwartz RB et al, Demirtas $\mathrm{O}$ et al and Junewar V et al. ${ }^{9,11,14}$ Poor innervations of the posterior cerebral vasculature make the occipital lobe more prone for PRES. PRES was a predominant neuroradiographic finding in present study which corroborated with findings of Sharma G et al. ${ }^{10}$ Although PRES is a pathognomic neuroimaging finding in subjects with hypertensive encephalopathy, atypical findings and atypical distribution of brain lesion may be seen on neuroimaging.

In the present study, 93.8\% subjects had headache and $34.5 \%$ subjects with headache had evidence of neurological involvement on MRI. Similarly in the study conducted by Demirtas O et al, $94.87 \%$ subjects had headache and nearly $50 \%$ of these had evidence of neurological involvement on cranial MRI. ${ }^{14}$ The headache is one of the warning neurological symptoms and the subjects with such symptoms were terminated at priority in order to halt the endothelial dysfunction and further neuropathology. In the present study fewer subjects (36.5\%) with premonitory visual symptoms had radiological evidence of neurological involvement on cranial MRI imaging. Whereas MRI findings have been reported in $71.4 \%$ subjects presenting with visual symptoms in the study conducted by Demirtas $\mathrm{O}$ et al and $94.4 \%$ subjects in the study conducted by Junewar $\mathrm{V}$ et al. ${ }^{9,14}$ The difference could be due to the fact that the more number of subjects had cranial MRI in the present study. The blurring of vision is one of the warning neurological symptoms and the subjects with such symptoms were terminated at priority in order to halt the endothelial dysfunction and further neuropathology.

Association has been observed between depression of consciousness, seizures and MRI findings in present study. This is well supported the findings of studies by Demirtas $\mathrm{O}$ et al and Junewar $\mathrm{V}$ et al respectively. ${ }^{9,14}$ It is proposed that direct endothelial dysfunction leading to an 
increase in the permeability of the blood-brain barrier may be the additional mechanism responsible for parenchymal fluid extravasation. Although the exact cause of the endothelial injury in patients with eclampsia is not known, circulating endothelial toxins or antibodies are thought to be responsible.

The mean SBP, DBP and MAP in the MRI positive subjects in the present study was comparable to the one observed by Junewar $\mathrm{V}$ et al. ${ }^{9}$ Clinical and radiological signs in patients with preeclampsia and eclampsia are believed to be related to the effect of increased systolic blood pressure on the auto regulation of the cerabral vasculature and is now believed to be the most commonly caused by vasogenicedema that arises from the escape of fluid from intravascular compartment into the interstitium because of breakthrough of auto regulation. ${ }^{15}$

In subjects with MRI findings haematocrit, LDH, uric acid and ApTT was significantly higher (pvalue 0.008$)$, (p-value 0.00$)$, (p-value 0.041 ) and (p-value 0.02 ) respectively than those without MR imaging findings. Similarly Demirtas $\mathrm{O}$ et al and Junewar $\mathrm{V}$ et al also observed significantly higher levels of LDH and uric acid, although haematocrit and ApTT did not differ in relation to MRI findings. They did not observe any difference in the mean thrombocyte count between MR imaging positive and negative subjects.

The mean platelet count in the present study was higher in MR imaging positive subjects, which was an unusual finding. Exclusion of subjects with DIC, HELLP and delay in neuroimaging could be the possible explanation as the patients had to be transported over a distance of 2 kilometers for neuroimaging.

The serum creatinine levels did not differ significantly in MRI positive and MRI negative subjects.

$16 / 23(69.5 \%$ ) subjects with neuroimaging findings had abnormal RBC morphology (p-value 0.00) Elevated LDH and abnormal RBC morphology are indicators of hemolysis. Elevated uric acid and serum creatinine are indicators of renal dysfunction. Endothelial injury yields to morphological disturbance in erythrocytes and microvascular hemorrhage. Therefore the brain lesions are found to be associated with high LDH levels, deranged renal functions and RBC morphology. These lab parameters are the predictors of abnormal radiological findings.

\section{Conclusion}

The cause of hypertensive disorders of pregnancy has not yet been explained. The brain lesions detected on MR imaging might shed light on pathogenesis of the disease. The vasogenicedema most prominently at the posterior region of cerebral hemisphere was detected on MRI in all eclamptic women subjected to neuroimaging. The cranial MRI was found to be $>90 \%$ sensitive for headache and visual disturbances and $100 \%$ specific for depression of consciousness and seizures. Cytotoxic edema and irreversible brain damage occur if etiological factor remains untreated.

Indicators of endothelial dysfunction i.e. abnormal RBC morphology, elevated LDH, deranged renal function tests were significantly associated with MRI positivity. Of all laboratory derangements the cranial MRI was found to have high sensitivity (91.30\%) for LDH and high specificity (100\%) for abnormal RBC morphology. We conclude that the brain edema in patients with preeclampsiaeclampsia syndrome is primarily associated with the laboratory based evidence of endothelial damage; more specific markers of endothelial dysfunction include fibronectin, tissue plasminogen activator, thrombomodulin, endothelin-1, and in particular Von willebrand factor. Measurement of the specific markers may be useful to evaluate endothelial integrity in patient who are preeclamptic, especially patient who are at risk of progression to hypertensive encephalopathy such as those with severe headache or other neurological signs and symptoms

Blood pressure parameters, although elevated in all patients, are not significantly different in those 
with or without brain edema, although the acute fluctuation of blood pressure may disrupt the cerebral autoregulatory mechanism and explains the association of neuroimaging findings with acutely high blood pressure.

Apart from diagnosing the brain involvement, neuroimaging in antenatals with severe hypertensive disorders would also be helpful in ruling out cerebrovascular accidents. Since the neuroimaging findings were observed in all eclamptic and disoriented women subjected to neuroimaging, it is recommend that such patients should be subjected to neuroimaging. Moreover, the patients with elevated BP parameters should also be subjected to neuroimaging in order to ensure timely intervention that may lower the morbidity and mortality among mothers with severe preeclampsia and eclampsia, which accounts for one tenth of all maternal deaths.

Funding: No funding sources

Conflict of interest: None declared

Ethical approval: Study was approved by the Institutional Ethics Committee

\section{References}

1. Chamotra S, Pathania K, Verma SK, Chaudhary A. Predicting pregnancy outcomes from homocysteine level: an evidence from a North Indian study. Int J Reprod Contracept Obstet Gynecol 2020; 9:1498-502.

2. Report of the National High Blood Pressure Education Program Working Group on high blood pressure in pregnancy. Am J Obstet Gynecol 2000; 183:S1-22

3. World Health Organization. WHO Recommendations for Prevention and Treatment of Pre-Eclampsia and Eclampsia. Geneva, Switzerland: World Health Organization; 2011

4. Patil Mithil M. Role of Neuroimaging in Patients with Atypical Eclampsia. J of
Obstet \& Gynecol of India 2012; 62 (5): 526-30

5. Roybart M, Seidman DS, Serr DM, Mashiach S. Review. Neurological Involvement in Hypertensive Disease of Pregnancy. Obstet \& gynaecol Survey 1991; 46(13): 656-64.

6. Diwan AG, Agrawal PH, Panchanadikar TM, Yadav SV, Joshi P. Late post-partum eclampsia with reversible encephalopathy syndrome: an interesting case. Indian $\mathrm{J}$ of Medical Specialities 2012

7. Chiou YH, Chen PH, Reversible Posterior Encephalopathy Syndrome as the Presentation of Late Post Partam Eclampsia: A case Report. Acta Neurologica Taiwanica 2007; 16 (3):15862.

8. Bartynski WS, Posterior Reversible Encephalopathy Syndrome, Part I: Fundamental Imaging and Clinical Features. Am J Neuroradiol 2008; 29: 1036-42.

9. Junewar V, Verma R, Sankhwar PL, Garg RK, Singh MK, Malhotra HS et al. Neuroimaging Features and Predictors of Outcome in Eclamptic Encephalopathy: A Prospective Observational Study. AJNR Am J Neuroradiol.2014; 35:1728-346

10. Sharma G, Joshi G, Purohit CR, Divyashree. Cranial MR Imaging in Eclampsia and Severe preeclampsia: A Prospective study. Journal of evolution of medical and dental sciences 2014;3(13):3250-59

11. Schwartz RB, Mulkern RV, Gudbjartsson $\mathrm{H}$ and Jolesz F: Diffusion-Weighted MR Imaging in Hypertensive Encephalopathy: Clues to Pathogenesis. AJNR Am J Neuroradiol 1998;19:859-62

12. Sharma G, Bhatt D, Yadav K, Agarwal S, Kochar S: Eclampsia Preeclampsia; Clinical and Neurological Correlates, IOSR Journal of Dental and Medical 
Sciences (IOSR-JDMS).Volume 15, Issue

5 Ver. III (May. 2016), PP 87-91

13. Dahiya K, Rathod M, Rohilla S, Dahiya P: Correlation between Neuroimaging and Clinical Presentation in Eclampsia, International Journal of Obstetrics and Gynaecology Research (IJOGR) Vol. 3 (2016) No.6, pp. 375-384.

14. Demirtas O, Gelal F, Vidinli BD, Demirtas LO, Uluc E, Baloglu A. Cranial MR Imaging with clinical correlation in preeclampsia and eclampsia. Diagn Interv Radiol 2005; 11: 189-94.

15. Swain S, Ohha KN, Prakash A. Maternal and perinatal mortality due to eclampsia. Indian Pediatr 1993 Jun; 30(6):771-73.

16. Richard BS, Steven KF, Joseph FP, De Girolami U, Lalia A, Beckner KM. Preeclampsia-Eclamsia: Clinical and Neuroradiographic Correlates and Insights into the Pathogenesis of Hypertensive Encephalopathy. Radilogy 2000; 217: 37.

17. Cunnighum FG, Leveno KJ, Bloom SL, Spong CY, Dashe JS, Hoffman BL. Williams obstetrics: Hypertensive disorders in pregnancy. $24^{\text {th }}$ edition. Newyork: McGraw-Hill Education; 2014. Chapter no. $10: 1508-6135$. 